\title{
Study of Oxygen Reduction Reaction in Sulfuric Acid on Thin Porous Electrodes Composed of Carbon and Platinum
}

\author{
Marcelo L. CAlEgaro, Joelma PEREZ, Auro A. TANAKA, Edson A. TiCiAnelli \\ and Ernesto R. GONZALEZ* \\ Received December 14, 1995 ; Accepted February 16, 1996
}

\begin{abstract}
In this work, the oxygen reduction reaction was studied in $0.5 \mathrm{M}$ sulfuric acid solutions on platinum dispersed on carbon with $10,20,30$, and $40 \% \mathrm{w} / \mathrm{w} \mathrm{Pt} / \mathrm{C}$ ratios, using the thin porous coating technique. Cyclic voltammetry was used to evaluate the $\mathrm{Pt}$ electrochemical active areas of the electrodes and steady-state polarization curves obtained with the rotating disk electrode technique were employed to study the oxygen reduction reaction. Cyclic voltammetry showed that the activity of the Pt particles with respect to the hydrogen adsorption/desorption process is dependent on the particle size. From the combination of the polarization data with the thin filmflooded agglomerate model it was possible to obtain the kinetic and mechanistic parameters for the oxygen reduction reaction, which resulted only dependent on the catalyst nature. It was observed that the mechanism and the kinetic behavior of the reaction were the same as those on gas diffusion electrodes or on smooth polycrystalline platinum. The only difference was found in the Tafel slopes of the polarization diagrams which double as a consequence of the porous nature of the dispersed catalyst electrodes.
\end{abstract}

\section{INTRODUCTION}

The study of the oxygen reduction reaction (ORR) on dispersed platinum on carbon catalysts has been the object of several publications for over 20 years, because of the importance of these materials for application in various kinds of fuel cells ${ }^{1)}$. In a previous work conducted in our laboratory ${ }^{2}$, this reaction was studied in $1.0 \mathrm{M} \mathrm{NaOH}$ solutions on thin porous coating rotating disk electrodes (TPC/RDE) prepared with $0,10,20$ and $30 \% \mathrm{w} / \mathrm{w} \mathrm{Pt} / \mathrm{C}$ catalysts. Cyclic voltammetry (CV) and steady state polarization techniques were employed in the experimental investigation. An alkaline medium was chosen because of the reduced interference of redox processes due to surface functional groups on the carbon substrate, compared with acid electrolytes. The steady state oxygen reduction polarization data were analyzed using the thin film/flooded agglomerate model of gas diffusion electrodes, as mathematically treated in the Cartesian system by Raistrick et. $\mathrm{al}^{3,4}$ ). The main conclusions of the previous work were:

(i) The cyclic voltammetric experiments showed that the area per gram of the platinum particles diminishes with the increase of the $\mathrm{Pt} / \mathrm{C}$ ratio in the supported catalysts, in agreement with the tendency

Instituto de Química de São Carlos - USP, Departamento de Físico Química

CP 780, São Carlos, SP, CEP 13560-970, Brazil

Key Words: dispersed electrocatalysts, oxygen reduction, electrode modeling, thin porous coating electrode. shown by published Transmission Electron Microscopy (TEM) results ${ }^{5}$. However, differences of one order of magnitude were found between the absolute values of the platinum areas. These differences were explained in terms of the several limitations of the TEM and CV techniques and it was concluded that cyclic voltammetry provides more consistent results for the evaluation of the $\mathrm{Pt}$ active area, when an electrochemical system is considered.

(ii) Analyses of the ORR polarization data obtained with the TPC/RDE with the thin film/floodedagglomerate model provided a simple and adequate way to evaluate the performance of the supported catalysts. The predicted effect of doubling of the Tafel slope of the ORR on the PU/C catalysts due to diffusional problems in the flooded agglomerate was confirmed experimentally.

(iii) The kinetic parameters obtained for the ORR on the several catalysts indicated that, in alkaline media and for the lower $\mathrm{Pt} / \mathrm{C}$ ratios, there is a strong participation of the carbon substrate in the catalysis of the reaction.

In this work, the oxygen reduction reaction was studied in $0.5 \mathrm{M}$ sulfuric acid solutions on platinum dispersed on carbon with $0,10,20,30$, and $40 \%$ w/w $\mathrm{Pt} / \mathrm{C}$ ratios, using the same techniques presented above. As in the previous work, the RDE results for the ORR on the TPC/RDE electrodes were analyzed in terms of the thin film/flooded agglomerate model of

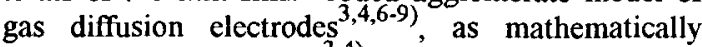
treated by Raistrick et. $\mathbf{a l}^{3,4}$ in the Cartesian system. 


\section{EXPERIMENTAL}

Although described previously for alkaline electrolyte ${ }^{2)}$, the details of the experimental working conditions will be presented here for consistency. Thin porous coating rotating disk electrodes were used for cyclic voltammetry and polarization measurements. These were prepared by mixing the catalyst powders [Vulcan XC-72 carbon, and 10, 20, 30 , and $40 \% \mathrm{w} / \mathrm{w} \mathrm{Pt} / \mathrm{carbon}$ (E-TEK Inc., USA)] with a dilute suspension $(\approx 2 \% \mathrm{w} / \mathrm{w})$ of a Teflon emulsion (DuPont TM30) and following the procedures described elsewhere ${ }^{10)}$.

A conventional three compartment electrochemical cell with Luggin capillary was used in the electrochemical experiments. A platinum foil and a saturated calomel electrode (SCE) served as counter and reference electrodes, respectively. In order to avoid contamination of the working electrode compartment, a salt bridge containing the working electrolyte was employed to connect the reference electrode to the cell. All the experiments were carried out in $0.5 \mathrm{M} \mathrm{H}_{2} \mathrm{SO}_{4}$ solutions, prepared from a high purity reagent (Merck) and water distilled and purified in a Milli-Q (Millipore) system. The electrolytes were saturated with purified $\mathrm{N}_{2}$ or $\mathrm{O}_{2}$ gases. All the experiments were conducted at room temperature $\left(25 \pm 1^{\circ} \mathrm{C}\right)$.

The cyclic voltammetry and rotating disk polarization measurements were performed with a PARC Model 273 potentiostat coupled with an IBMPC compatible microcomputer. The electrode rotation speeds were controlled by a AFMSRE/ASR Pine Instrument Co. system. All the voltammetric curves reported in this paper correspond essentially to the stationary responses obtained on electrodes cycled between -0.25 to $1.15 \mathrm{~V}$ vs. SCE, that is, between the onset of hydrogen evolution and the start of carbon corrosion/oxygen evolution. Furthermore, several potential scan rates were employed to record the cyclic voltammograms, but only the responses recorded at 5 $\mathrm{mV} \mathrm{s}^{-1}$ were considered for quantitative analyses. The Pt active areas were evaluated from the voltammetric curves by assuming the value of $210 \mu \mathrm{C} \mathrm{cm}^{-2}$ for the hydrogen desorption charge on smooth Pt electrodes ${ }^{5)}$ and taking into account the weight of the TPC layers obtained after performing the electrochemical measurements. The oxygen reduction polarization curves involving the TPC were recorded point-bypoint in the potentiostatic mode to avoid the high capacitive currents associated with this type of electrode. The electrodes were polarized in both directions (increasing or decreasing the polarization) until a stable performance was observed, but only the polarization curves obtained at increasing polarizations are presented.

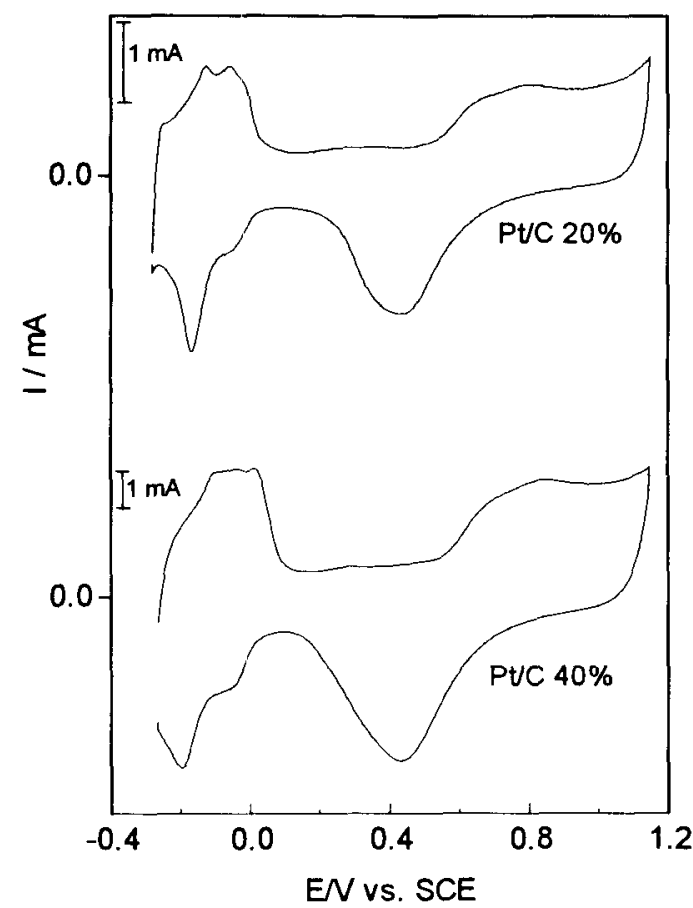

Fig. 1 Cyclic voltamograms of TPC electrodes prepared with different $\mathrm{Pt} / \mathrm{C}$ catalysts in $\mathrm{N}_{2}$-saturated $0.5 \mathrm{M} \mathrm{H}_{2} \mathrm{SO}_{4}$ solution. $\mathrm{v}=5 \mathrm{mV} / \mathrm{s}$.

\section{RESULTS AND DATA PROCESSING}

Figure 1 shows the cyclic voltammograms in $0.5 \mathrm{M} \mathrm{H}_{2} \mathrm{SO}_{4}$ solution for the 20 and $40 \mathrm{wt} . \% \mathrm{Pt} / \mathrm{C}$. In contrast with the voltammograms obtained employing gas diffusion electrodes ${ }^{11}$ in acid media, a quite reasonable resolution of the platinum features was found. For the other catalysts the voltammograms presented the same qualitative features. Table 1 presents the active area per gram of platinum on the TPC electrodes, evaluated from the cyclic voltammetric responses following the procedure described before ${ }^{2)}$. For comparison, the corresponding results obtained previously using TEM $^{\text {s) }}$ are also included.

Figure 2 presents the oxygen reduction polarization curves for several rotation rates obtained in $0.5 \mathrm{M} \mathrm{H}_{2} \mathrm{SO}_{4}$ on the TPC/RDE electrode prepared with 20 wt.\% Pt/C catalyst. Figure 3 illustrates the

Table 1 Active platinum surface areas obtained from the cyclic voltammetric experiments in acid solutions.

\begin{tabular}{lccc}
\hline & \multicolumn{2}{c}{ Active Area } & Area/TEM \\
\cline { 2 - 3 } Electrode & $\mathrm{cm}^{2}$ & $\left(\mathrm{~m}^{2} / \mathrm{g} \mathrm{Pt}\right)$ & $\left(\mathrm{m}^{2} / \mathrm{g} \mathrm{Pt}\right)$ \\
\hline $10 \% \mathrm{Pt} / \mathrm{C}$ & 93 & 47 & 135 \\
$20 \% \mathrm{Pt} / \mathrm{C}$ & 215 & 47 & 100 \\
$30 \% \mathrm{PtC}$ & 240 & 36 & $88^{(\mathrm{a})}$ \\
$40 \% \mathrm{Pt} / \mathrm{C}$ & 366 & 35 & 80 \\
\hline (a) E-TEK, Inc. 1995. Catalogue specification.
\end{tabular}




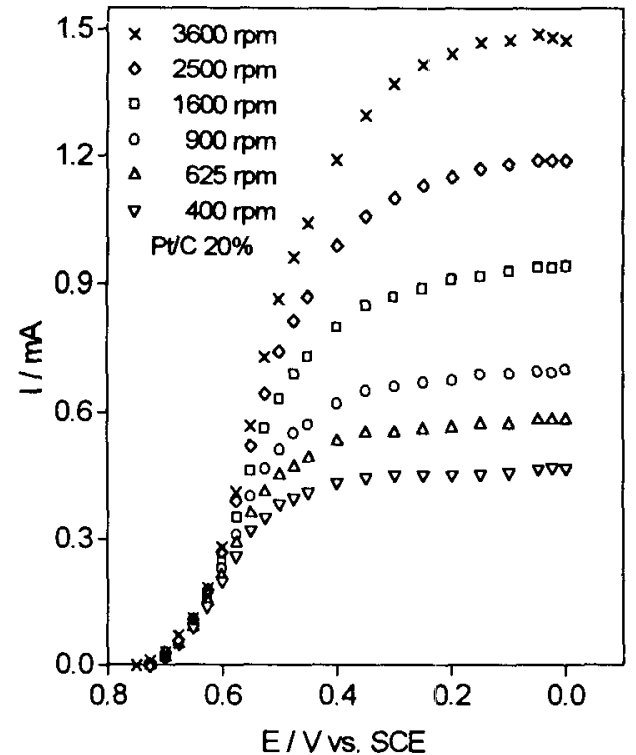

Fig. 2 Steady-state polarization curves at different electrode rotation rates for oxygen reduction on $20 \%$ $\mathrm{Pt} / \mathrm{C}$ TPC-RDE in $0.5 \mathrm{M} \mathrm{H}_{2} \mathrm{SO}_{4}$ solution.

effect of catalyst composition for a constant rotation rate of the electrode $(1600 \mathrm{rpm})$. To compare the specific performance of the catalysts with respect to the ORR, the values of current obtained at $0.6 \mathrm{~V}$ in the polarization curves were normalized dividing by the surface area of platinum in the electrodes, using the TEM and $\mathrm{CV}$ values. The results of such calculations are presented in Table 2.

The RDE results for the ORR on the TPC electrodes were analyzed in terms of the thin filmflooded agglomerate model originally developed for gas diffusion electrodes, ${ }^{3,4}$. The several aspects stressing the correspondence between the thin film/flooded agglomerate model and the TPC rotating disk electrode were presented before ${ }^{2)}$. According to this theoretical model ${ }^{2-4}$, the reduced current density (J) passing from the agglomerate into the solution can be written as:

$$
J=i / i_{0}=\frac{\exp (\mu) \frac{\tanh [\phi \exp (\mu / 2)]}{\phi \exp (\mu / 2)}}{1+\Gamma \exp (\mu) \frac{\tanh [\phi \exp (\mu / 2)]}{\phi \exp (\mu / 2)}}
$$

where $i$ is the actual current density, $i_{0}$ is the exchange current density $\left(\mathrm{nFk}^{\circ} \mathrm{C}^{*}\right), \mu$ is the reduced overpotential $(\eta / b), \phi$ is the dc potential-independent agglomerate diffusion parameter $\left(\left(\mathrm{k}^{\circ} \mathrm{L}_{\mathrm{y}} / \mathrm{D}_{\mathrm{a}}\right)^{1 / 2}\right), \Gamma$ is the thin film diffusion parameter $\left(\delta \mathrm{k}^{\circ} / \mathrm{D}_{f}\right)$, $\mathrm{n}$ is the number of electrons transferred, $k^{\circ}$ is the effective rate

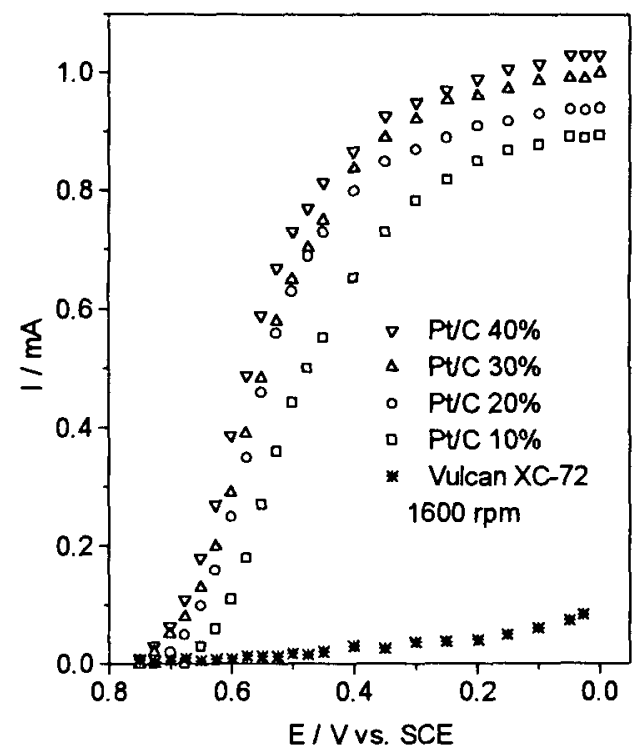

Fig. 3 Steady-state polarization curves for oxygen reduction on TPC-RDE prepared with different Pt/C catalysts in $0.5 \mathrm{M} \mathrm{H}_{2} \mathrm{SO}_{4}$ solution. $f=1600 \mathrm{rpm}$.

constant at the reversible potential $\left(k^{\prime} A_{v} L_{y}\right), k^{\prime}$ is the rate constant on the catalyst at the reversible potential, $A_{v}$ is the catalyst area per unit volume, $C^{*}$ is the bulk reactant concentration, $b$ is the Tafel slope of the ORR in the catalyst, $D_{a}$ is the diffusion coefficient of oxygen in the agglomerate, and $D_{f}$ is the diffusion coefficient of oxygen in the thin film.

Two limiting ${ }^{3,4)}$ structural effects are predicted from the analyses of Eq. (1) for the polarization behavior of the TPC electrode: (i) large values of $\phi$ (large $L_{y}$ ) lead to a duplication of the slope of the $\mu$ vs. ln J plot at larger values of J (or $\mu$ ); (ii) large values of $\Gamma$ (large $\delta$ ) lead to the appearance of limiting diffusional current in the plot.

Substitution of $\mu, \phi$, and $\Gamma$ in Eq. (1) gives the theoretical dependence of the current density as a function of the electrode potential and the several physicochemical and electrochemical parameters of the system: $D_{a}, D_{f}, L_{y}, \delta, C^{*}, i_{o}, b$ and $n$.

Parametrization of the theoretical equation was performed by using the depth value of the electrode cavity, $\mathrm{L}_{\mathrm{y}} \cong 0.034 \mathrm{~cm}$, measured with a digital micrometer, tabulated values for the diffusion coefficient and solubility of oxygen in the electrolyte ${ }^{12)}: D_{\mathrm{f}}=1.5 \times 10^{-5} \mathrm{~cm}^{2} \mathrm{~s}^{-1}, \mathrm{C}^{*}=1.1 \times 10^{-6}$ mole $\mathrm{cm}^{-3}$, respectively, and values of the thin film diffusion parameter obtained from RDE equations ${ }^{13}$, $\delta=3.0 \times 10^{-3}, 2.4 \times 10^{-3}, 2.0 \times 10^{-3}, 1.5 \times 10^{-3}, 1.2 \times$ $10^{-3}, 1.0 \times 10^{-3} \mathrm{~cm}$ for rotation rates $400,625,900$, 1600,2500 , and $3600 \mathrm{rpm}$, respectively. The value of 
Table 2 Specific current densities for the ORR for the several catalysts at an electrode potential of $0.6 \mathrm{~V}$. The areas of $\mathrm{Pt}$ were those presented in Table 1 for the TEM and CV results.

\begin{tabular}{cccc}
\hline $\begin{array}{c}\text { Electrode } \\
\text { (wt.\% Pt/C) }\end{array}$ & $\begin{array}{c}\text { Current } \\
\text { at 0.6 V } \\
(\mathrm{mA})\end{array}$ & $\begin{array}{c}\text { Current density per } \\
\text { unit area of Pt } \\
\left.(\mathrm{mA} \mathrm{cm})^{-2}\right)\end{array}$ \\
\hline 10 & 0.11 & $4.1 \times 10^{-4}$ & $\mathrm{CV}$ \\
\hline 20 & 0.25 & $5.4 \times 10^{-3}$ & $1.2 \times 10^{-3}$ \\
30 & 0.29 & $4.9 \times 10^{-4}$ & $1.2 \times 10^{-3}$ \\
40 & 0.39 & $4.7 \times 10^{-4}$ & $1.1 \times 10^{-3}$ \\
\hline
\end{tabular}

$D_{a}$, the diffusion coefficient in the flooded agglomerate, was assumed to be the same as in the bulk electrolyte, that is, $D_{a} \cong D_{f}$. This approximation did not introduce major changes in the results since small variations in $D_{a}$ were evaluated to have negligible effect on the simulated curves.

Iteration to obtain the best values of $i_{0}, b$, and $n$ is necessary and it was executed considering that the Tafel slope of the ORR is dependent on the coverage of oxygen on the platinum surface which follows a Temkin isotherm at high electrode potentials $(>-0.55 \mathrm{~V}$ ), and a Langmuir isotherm at lower electrode potentials $^{14)}(<\sim 0.55 \mathrm{~V})$. Figures 4 and 5 show examples of the application of Eq. (1) to the experimental results, indicating the very good quality of the fittings. The average (for all rotation rates) values of $i_{o}, b$, and $n$ obtained from these fittings for the ORR in the several catalysts are presented in Table 3 for the two conditions of oxygen coverage.

Finally, the polarization data were analyzed in terms of mass transport corrected Tafel diagrams obtained by plotting the experimental data as $E$ vs. log [(i $\left.\left.x i_{d}\right) /\left(i_{d}-i\right)\right]$, where $i_{d}$ is the limiting current density ${ }^{13}$, and simulating the theoretical lines using the appropriated parametrization for each situation and taking the thin film diffusion parameter $\Gamma=0$ in Eq. (1). Examples of such Tafel plots are presented in Figure 6.

\section{DISCUSSION}

The results in Table 1 show that the total CV area increases with the increase of the $\mathrm{Pt} / \mathrm{C}$ weight ratio. The same behavior can be observed from the $i_{0}$ values presented in Table 3 . Both facts are related to the increase of the Pt loading on the TPC electrodes with the increase of $\mathrm{Pt} / \mathrm{C}$ ratio, since the cavity is filled with an approximately constant volume of the materials to form the active layer. A more close correlation between the predictions of $\mathrm{CV}$ and the values of $i_{o}$ cannot be established because of the large uncertainty in the absolute values of $i_{o}$ introduced by the modeling analysis and by the extrapolation to reach the ORR standard potential.
On the other hand, it can be observed in Table 1 that the CV surface area per gram of platinum presents a tendency to decrease with the increase of the $\mathrm{Pt} / \mathrm{C}$ ratio. As also observed in alkaline media ${ }^{2}$ ) and from the TEM results), this effect can be attributed to an increase of the Pt particle size with the increase of $\mathrm{Pt} / \mathrm{C}$ ratio. However, it is observed that the CV values of specific area are ca. $40 \%$ of those obtained with TEM. Since the electrode is completely filled with the electrolyte, the reasons for these discrepancies can be discussed in terms of the several limitations that the CV and TEM techniques present for the determination of the $\mathrm{Pt}$ area ${ }^{2}$. For cyclic voltammetry, the limiting factors may be: (i) imperfect electric contact between the catalyst particles, which gives underestimated currents in the recorded cyclic voltammograms and (ii) the presence of impurities, leading to a partial blocking of the hydrogen adsorption/desorption process. For the TEM technique, the following limitations can be considered: (i) it is assumed a spherical geometry for the platinum particles, which is a rough approximation for the real system and (ii) the total area of $\mathrm{Pt}$ loaded on the carbon is considered in the calculation, therefore, including the area partially blocked as a consequence of anchoring the Pt on the carbon substrate, the area of $\mathrm{Pt}$ which is not in contact with the carbon particles and also the area of a large amount of too small or too large platinum particles which may present reduced voltammetric activity.

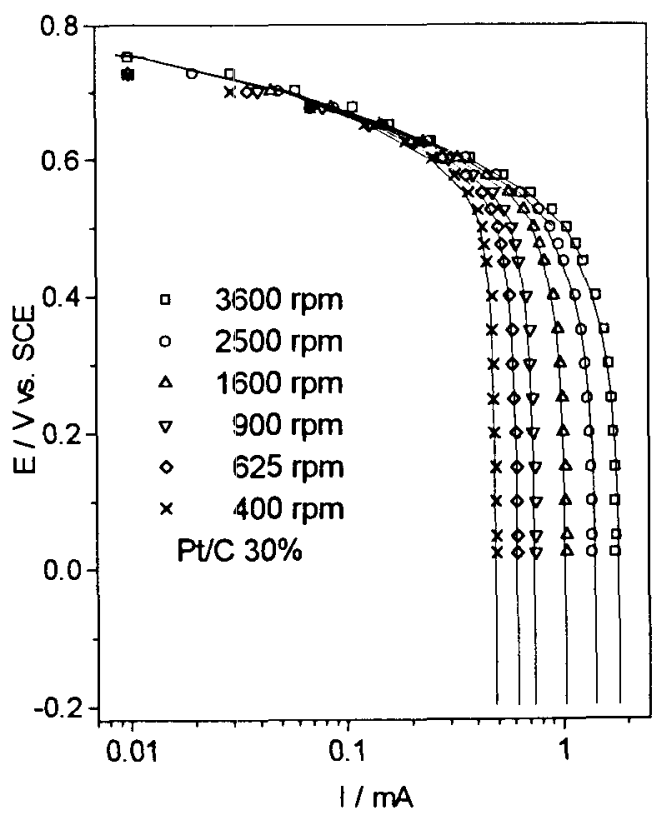

Fig. 4 Experimental (symbols) and simulated (solid lines) Tafel plots for oxygen reduction on $30 \% \mathrm{Pt} / \mathrm{C}$ electrode in $0.5 \mathrm{M} \mathrm{H}_{2} \mathrm{SO}_{4}$ solution for different electrode rotation rates. 




Fig. 5 Experimental (symbols) and simulated (solid lines) Tafel plots for oxygen reduction on different $\mathrm{Pt} / \mathrm{C}$ catalysts in $0.5 \mathrm{M} \mathrm{H}_{2} \mathrm{SO}_{4}$ solution. $f=1600 \mathrm{mpm}$.

This last point has been used to explain the change of the specific activity of the platinum crystallites with respect to the ORR, as a function of the particle size which reaches a maximum for particle sizes close to $2-3 \mathrm{~nm}^{15,16)}$. In the present case, this corresponds to the catalyst with $20 \mathrm{wt} . \% \mathrm{P} / \mathrm{C}^{5}$. The results in Table 2 show that the specific current density obtained by normalizing the current at $0.6 \mathrm{~V}$ using the TEM Pt area also reaches a maximum for this catalyst, indicating that the same tendency proposed previously ${ }^{15}$ ) for the catalyst activity is observed here when the TEM surface area is considered.

On the other hand, the results of Table 2 show that the specific activity is independent of the catalyst (or particle size) when the current is normalized using the CV area. This is a very important observation since it implies that the same tendency found in the activity for the ORR with respect to particle size, holds for the hydrogen adsorption/desorption process in the cyclic voltammetry.

The polarization diagrams presented in Figure 3 clearly show the very low activity of the pure carbon catalyst for the ORR. Koutecky-Levich plots $\left(i^{-1}\right.$ vs. $\mathrm{f}^{1 / 2}$ ) constructed from the experimental polarization data at several electrode potentials present straight parallel lines for all $\mathrm{Pt} / \mathrm{C}$ catalysts. This behavior is an indication that the ORR is first order with respect to the oxygen concentration and the number of electrons

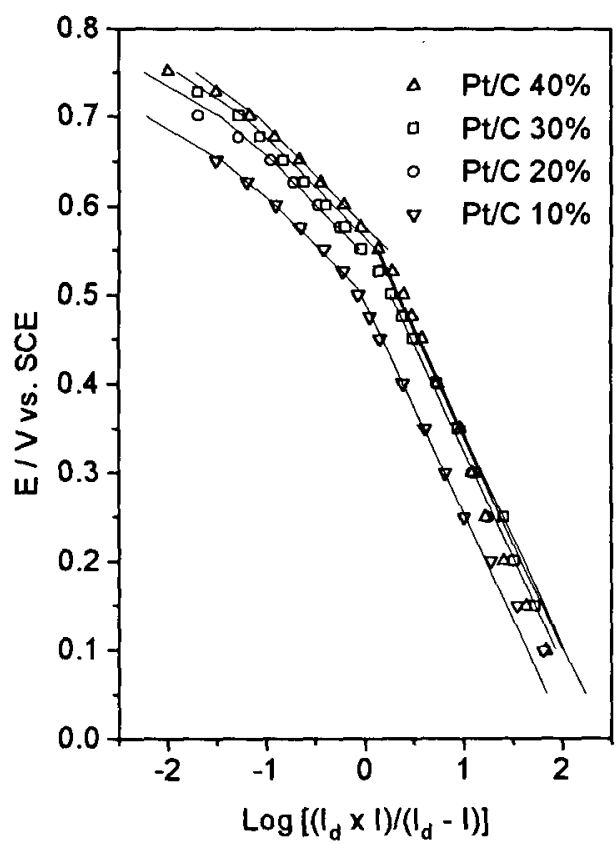

Fig. 6 Mass-transport corrected Tafel plots for oxygen reduction on different $\mathrm{Pt} / \mathrm{C}$ catalysts in $0.5 \mathrm{M} \mathrm{H}_{2} \mathrm{SO}_{4}$ solution. $f=1600 \mathrm{rpm}$. Solid line: simulated Tafel plot with $\Gamma=0$ in Eq. 1 .

involved in the reaction is constant, independent of the electrode potential and of the catalyst $\mathrm{Pt} / \mathrm{C}$ composition. Taking the slopes of such lines and using the values of $\mathrm{D}$ and $\mathrm{C}^{*}$ presented before, $v^{12)}=$ $1.2 \times 10^{-2} \mathrm{~cm} \mathrm{~s}^{-1}, \mathrm{~F}=96,487 \mathrm{C} \mathrm{mol}^{-1}$, and $\mathrm{A}=$ $0.196 \mathrm{~cm}^{2}$ (geometric area) in the Koutecký-Levich equations ${ }^{13)}$, the value of $n$ resulted close to 4 for all catalysts. The results of Table 3 also show a value of $n=4$ obtained from the fitting of the theoretical thin film-flooded agglomerate equation for the ORR in all catalysts. Thus, in this respect, the behavior of the dispersed catalysts with the several $\mathrm{Pt} / \mathrm{C}$ ratios in the TPC electrodes is the same as that for the ORR in acid media with smooth platinum ${ }^{1,12,14,17)}$ or with gas diffusion electrodes ${ }^{11,18)}$.

Table 3 shows that the Tafel slopes obtained from the fittings have the values expected for the ORR on the platinum catalyst. For smooth polycrystalline platinum and gas diffusion electrodes, two Tafel regions, with slopes of 60 and $120 \mathrm{mV} \mathrm{deca}^{-1}$ (at $25^{\circ} \mathrm{C}$ ) for low and high overpotentials, respectively, have been observed for the ORR in acid solutions. The existence of two slopes is explained in terms of the coverage of the electrode surface by adsorbed oxygen, which follows a Temkin isotherm at low overpotentials and a Langmuir isotherm at higher overpotentials $^{14}$ ). The changes of adsorption conditions can be better understood using the cyclic voltammograms obtained in the absence of dissolved 
Table 3 Fitting parameters obtained for oxygen reduction on Pt/C catalysts in $0.5 \mathrm{M} \mathrm{H}_{2} \mathrm{SO}_{4}$ at $25^{\circ} \mathrm{C}$.

\begin{tabular}{cccccc}
\hline & \multicolumn{2}{c}{$\begin{array}{c}\text { Low current } \\
\text { density }\end{array}$} & \multicolumn{2}{c}{$\begin{array}{c}\text { High current } \\
\text { density }\end{array}$} & \\
\cline { 2 - 5 } $\mathrm{P} / \mathrm{C}$ & $\begin{array}{c}\mathrm{i}_{0} \\
\mathrm{~b}(\mathrm{mV}\end{array}$ & $\begin{array}{c}\mathrm{i}_{0}(\mathrm{~A} \\
\mathrm{b}(\mathrm{mV}\end{array}$ & $\mathbf{n}$ \\
$\left.\mathrm{cm}^{-2}\right)$ & $\begin{array}{c}\left(\mathrm{A} \mathrm{cm}^{-2}\right) \\
\left.\mathrm{deca}^{-1}\right)\end{array}$ & $\left.\mathrm{cm}^{-2}\right)$ & deca $\left.^{-1}\right)$ & \\
\hline 10 & $5 \times 10^{-11}$ & 56 & $24 \times 10^{-6}$ & 120 & 4 \\
20 & $4 \times 10^{-10}$ & 57 & $74 \times 10^{-6}$ & 120 & 4 \\
30 & $9 \times 10^{-10}$ & 57 & $94 \times 10^{-6}$ & 120 & 4.1 \\
40 & $15 \times 10^{-10}$ & 57 & $14 \times 10^{-5}$ & 120 & 4.1 \\
\hline
\end{tabular}

oxygen (Figure 1). In the region above ca. $0.55 \mathrm{~V}$ vs. $\mathrm{SCE}$, the platinum surface is covered with adsorbed oxygenated species, while below this value the oxides are reduced. Experimentally, it has been observed that the ORR presents Tafel slopes of $60 \mathrm{mV} \mathrm{deca-1} \mathrm{on} \mathrm{the}$ oxide covered $\mathrm{Pt}$ region and $120 \mathrm{mV} \mathrm{deca}{ }^{-1}$ on the oxide-free region ${ }^{14}$.

It is important to observe that the above values of Tafel slopes could be obtained only with the support of the modeling analysis of experimental data. It is seen that the experimental points of the Tafel plots presented in Figure 6 fit the theoretical line with a slope of $60 \mathrm{mV}$ deca ${ }^{-1}$ only for a few points located in the highest voltage region of the diagram. At lower potentials, a region with a $120 \mathrm{mV} \mathrm{deca}{ }^{-1}$ slope is defined, and then a $240 \mathrm{mV}$ deca $^{-1}$ slope is clearly seen. According to the model, the intermediate region with a slope of $120 \mathrm{mV} \mathrm{deca-1}$ arises as a consequence of structural effects in the electrodes which lead to the doubling of the effective Tafel slope of the reaction on the considered catalyst in this range of potential. This behavior is consistent with the cyclic voltammograms of Figure 1, since the $120 \mathrm{mV} \mathrm{deca}^{-1}$ slope appears in a region of potential in which the catalyst is covered to a large extent with oxide. Following this interpretation, the $240 \mathrm{mV}$ deca $^{-1}$ Tafel slope observed in Figure 6 corresponds to the doubling of the effective value $\left(120 \mathrm{mV} \mathrm{deca}^{-1}\right)$ in the oxide free region.

\section{CONCLUSIONS}

Taking into account the behavior of the ORR on the dispersed catalysts, several important observations can be made from the results of the present work:

(i) As in alkaline media ${ }^{2}$, cyclic voltammetry showed that the measured area of the platinum catalysts diminishes with the increase of the $\mathrm{Pt} / \mathrm{C}$ ratio. However, the results in acid media show that this trend can be related to the specific activity of the dispersed platinum crystallites with respect to the hydrogen adsorption/desorption process which is dependent on the particle size. In this sense the activity for hydrogen adsorption/desorption is similar to that observed for the specific activity of the platinum crystallites for the ORR, which reaches a maximum for particle sizes close to $2-3 \mathrm{~nm}^{15}$. (ii) The polarization curves and the kinetic parameters obtained for the ORR on the several catalysts show a negligible participation of the carbon substrate in the catalysis of the reaction in acid solution. This is in contrast with the results in alkaline media ${ }^{2}$, where the participation of carbon through a $2 e^{-}$pathway was clearly observed;

(iii) As in alkaline media ${ }^{2)}$, analyses of the ORR polarization data obtained with the TPC-RDE with the thin film/flooded-agglomerate model provided a simple and adequate way to evaluate the performance of the supported catalysts. From this analysis it was possible to obtain the real Tafel slopes of the ORR in the materials which resulted $60 \mathrm{mV} \mathrm{deca}^{-1}$ for the oxide covered electrode and $120 \mathrm{mV} \mathrm{deca}{ }^{-1}$ for the oxide free surface. As predicted by the model the doubling of the above Tafel slopes due to the structural influence of the porous electrodes is clearly observed.

The authors wish to thank Fundação de Amparo à Pesquisa do Estado de São Paulo (FAPESP), Conselho Nacional de Desenvolvimento Científico e Tecnológico (CNPq) and Financiadora de Estudos e Projetos (FINEP), for financial supports.

\section{REFERENCES}

1) R. Tarasevich, A. Sadkowski and E. Yeager; in B. E. Conway, J.O'M. Bockris, E. Yeager, S. U. M. Khan and R. White (eds), "Comprehensive Treatise of Electrochemistry", Vol. 7, Plenum Press, New York, pg. 301, 1983.

2) J. Perez, A. A. Tanaka, E. R. Gonzalez and E. A. Ticianelli, J. Electrochem. Soc., 141, 431 (1994).

3) T. E. Springer and I. D. Raistrick, J. Electrochem. Soc., 136, 1594 (1989).

4) I. D. Raistrick, Electrochim. Acta, 35, 1579 (1990).

5) E. A. Ticianelli, J. G. Beery and S. Srinivasan, $J$. Applied Electrochem., 21, 597 (1991).

6) J. Giner and C. Hunter, J. Electrochem. Soc., 116, 1124 (1969).

7) M. B. Cutlip, Electrochim. Acta, 20767 (1975).

8) R. P. Iczkowiski and M. B. Cutlip, J. Electrochem. Soc, 127,1433 (1980)

9) P. Bjornbom, Electrochim Acta, 32, 115 (1987).

10)A. A. Tanaka, C. Fierro, D. Scherson, and E. B. Yeager, J. Phys. Chem., 91, 3799 (1987).

11)S. L. A. da Silva, E. A. Ticianelli, J. Electroanal. Chem., 391, 101 (1995).

12)J. B. Floriano, E. A. Ticianelli and E. R. Gonzalez, J. Electroanal. Chem.,367, 157 (1994).

13)A. J. Bard and L. R. Faulkner, Electrochemical Methods, John Wiley, New York, 1980.

14)D. B. Sepa, M. V. Vojnovic and A. Damjanovic, Electrochim. Acta, 26, 781 (1981).

15)K. Kinoshita, J. Electrochem. Soc., 137, 845 (1990) 
16)T. E. Springer, M. S. Wilson, F. Garzon, T. A. Zawodzinski and S. Gottesfeld, in "Proceedings of the Symposium on Batteries and Fuel Cells for Stationary and Electric Vehicle Applications", A. R. Landgrebe and Z. Takehara (eds), PV 93-8, p. 258, The Electrochemical Society Proceeding Series, Pennington, NJ (1993).
17) A. Parthasarathy, S. Srinivasan, A. J. Appleby and C. R. Martin, J. Electrochem. Soc., 139, 2856 (1992)

18)D. R. de Sena, E. A. Ticianelli and E. R. Gonzalez, J. Electroanal. Chem., 357, 225 (1993). 